\title{
Generic feature of future crossing of phantom divide in viable $f(R)$ gravity models
}

\author{
Kazuharu Bamba*, Chao-Qiang Geng ${ }^{\dagger}$ and Chung-Chi Lee ${ }^{\ddagger}$ \\ Department of Physics, National Tsing Hua University, Hsinchu, Taiwan 300
}

\begin{abstract}
We study the equation of state for dark energy and explicitly demonstrate that the future crossings of the phantom divide line $w_{\mathrm{DE}}=-1$ are the generic feature in the existing viable $f(R)$ gravity models. We also explore the future evolution of the cosmological horizon entropy and illustrate that the cosmological horizon entropy oscillates with time due to the oscillatory behavior of the Hubble parameter. The important cosmological consequence is that in the future, the sign of the time derivative of the Hubble parameter changes from negative to positive in these viable $f(R)$ gravity models.

PACS numbers: $04.50 . K d, 95.36 .+\mathrm{x}, 98.80 .-\mathrm{k}$
\end{abstract}

\footnotetext{
* E-mail address: bamba@phys.nthu.edu.tw

$\dagger$ E-mail address: geng@phys.nthu.edu.tw

$\ddagger$ E-mail address: g9522545@oz.nthu.edu.tw
} 


\section{INTRODUCTION}

The cosmological observations such as supernovae Ia [1], cosmic microwave background radiation [2], large scale structure [3], and weak lensing [4] have revealed that the universe has been undergoing an accelerating expansion since the recent "past", which is one of the most challenging problems in physics today. There are two representative approaches to explain the late time acceleration of the universe. One is the introduction of "dark energy" in the framework of general relativity [5]. The other is the consideration of a modified gravitational theory, such as $f(R)$ gravity [6-8]. In this study, we will concentrate on the later approach.

It has been commonly adopted that a viable $f(R)$ gravity model needs to satisfy the following conditions: (a) positivity of the effective gravitational coupling, (b) stability of cosmological perturbations [9], (c) asymptotic behavior to the standard $\Lambda$-Cold-DarkMatter $(\Lambda \mathrm{CDM})$ model in the large curvature regime, $(\mathrm{d})$ stability of the late-time de Sitter point [10], (e) constraints from the equivalence principle, and (f) solar-system constraints [11]. Several viable models have been constructed in the literature, such as the popular ones: (i) Hu-Sawicki [12], (ii) Starobinsky [13], (iii) Tsujikawa [14], and (iv) the exponential gravity [15 18] models with the explicit forms shown in Table I. For other viable

TABLE I: Explicit forms of $f(R)$ in (i) Hu-Sawicki, (ii) Starobinsky, (iii) Tsujikawa, and (iv) the exponential gravity models.

\begin{tabular}{|c||c|c|}
\hline model & $f(R)$ & Constant parameters \\
\hline \hline (i) & $R-\frac{c_{1} R_{\mathrm{HS}}\left(R / R_{\mathrm{HS}}\right)^{p}}{c_{2}\left(R / R_{\mathrm{HS}}\right)^{p}+1}$ & $c_{1}, c_{2}, p(>0), R_{\mathrm{HS}}(>0)$ \\
\hline (ii) & $R+\lambda R_{\mathrm{S}}\left[\left(1+\frac{R^{2}}{R_{\mathrm{S}}^{2}}\right)^{-n}-1\right]$ & $\lambda(>0), n(>0), R_{\mathrm{S}}$ \\
\hline (iii) & $R-\mu R_{\mathrm{T}} \tanh \left(\frac{R}{R_{\mathrm{T}}}\right)$ & $\mu(>0), R_{\mathrm{T}}(>0)$ \\
\hline (iv) & $R-\beta R_{\mathrm{E}}\left(1-e^{-R / R_{\mathrm{E}}}\right)$ & $\beta, R_{\mathrm{E}}$ \\
\hline
\end{tabular}

models (e.g., models in Ref. [19]) and references, see a recent review in Ref. [8].

Recently, the cosmological observational data [20] also seems to indicate the crossing of the phantom divide $w_{\mathrm{DE}}=-1$ of the equation of state for dark energy in the near "past". To understand such a crossing, many attempts have been made. The most noticeable one is to use a phantom field with a negative kinetic energy term [21]. Clearly, it surfers a 
serious problem as it is not stable at the quantum level. On the other hand, the crossing of the phantom divide can also be realized in the above viable $f(R)$ models [12, 17, 18, 22] without violating any stability conditions. This is probably the most peculiar character of the modified gravitational models. Other $f(R)$ gravity models with realizing a crossing [6, 23] as well as multiple crossings [24] of the phantom boundary have also been examined.

However, most of the studies in $f(R)$ gravity have been focused on the past. In this paper, we would like to explore the future behaviors of the universe. In particular, we show that the viable $f(R)$ models generally exhibit the crossings of the phantom divide in the "future" too. In addition, we investigate the future evolution of the cosmological horizon entropy and demonstrate that the cosmological horizon entropy oscillates with time due to the oscillation of the Hubble parameter.

We use units of $k_{\mathrm{B}}=c=\hbar=1$ and denote the gravitational constant $8 \pi G$ by $\kappa^{2} \equiv$ $8 \pi / M_{\mathrm{Pl}}^{2}$ with the Planck mass of $M_{\mathrm{Pl}}=G^{-1 / 2}=1.2 \times 10^{19} \mathrm{GeV}$. We assume the flat Friedmann-Lemaître-Robertson-Walker (FLRW) space-time with the metric,

$$
d s^{2}=-d t^{2}+a^{2}(t) d \boldsymbol{x}^{2}
$$

where $a(t)$ is the scale factor.

The paper is organized as follows. In Sec. II, we explain $f(R)$ gravity and derive the gravitational field equations. We investigate the cosmological evolution in Sec. III. Finally, conclusions are given in Sec. IV.

\section{II. $f(R)$ GRAVITY}

The action of $f(R)$ gravity with matter is given by

$$
I=\int d^{4} x \sqrt{-g} \frac{f(R)}{2 \kappa^{2}}+I_{\text {matter }}\left(g_{\mu \nu}, \Upsilon_{\text {matter }}\right)
$$

where $g$ is the determinant of the metric tensor $g_{\mu \nu}, I_{\text {matter }}$ is the action of matter which is assumed to be minimally coupled to gravity, i.e., the action $I$ is written in the Jordan frame,

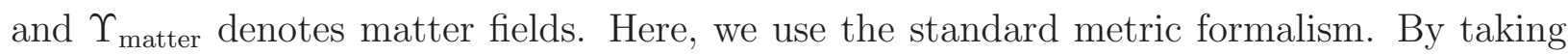
the variation of the action in Eq. (2) with respect to $g_{\mu \nu}$, one obtains [7]

$$
F G_{\mu \nu}=\kappa^{2} T_{\mu \nu}^{(\text {matter })}-\frac{1}{2} g_{\mu \nu}(F R-f)+\nabla_{\mu} \nabla_{\nu} F-g_{\mu \nu} \square F
$$


where $G_{\mu \nu}=R_{\mu \nu}-(1 / 2) g_{\mu \nu} R$ is the Einstein tensor, $F(R) \equiv d f(R) / d R, \nabla_{\mu}$ is the covariant derivative operator associated with $g_{\mu \nu}, \square \equiv g^{\mu \nu} \nabla_{\mu} \nabla_{\nu}$ is the covariant d'Alembertian for a scalar field, and $T_{\mu \nu}^{\text {(matter) }}$ is the contribution to the energy-momentum tensor from all perfect fluids of matter. From Eq. (3), we obtain the following gravitational field equations:

$$
\begin{aligned}
3 F H^{2} & =\kappa^{2} \rho_{\mathrm{M}}+\frac{1}{2}(F R-f)-3 H \dot{F}, \\
-2 F \dot{H} & =\kappa^{2}\left(\rho_{\mathrm{M}}+P_{\mathrm{M}}\right)+\ddot{F}-H \dot{F},
\end{aligned}
$$

where $H=\dot{a} / a$ is the Hubble parameter, the dot denotes the time derivative of $\partial / \partial t$, and $\rho_{\mathrm{M}}$ and $P_{\mathrm{M}}$ are the energy density and pressure of all perfect fluids of matter, respectively.

\section{COSMOLOGICAL EVOLUTION}

We analyze the cosmological evolution of dark energy including those of non-relativistic matter (cold dark matter and baryon) and radiation by solving Eq. (4) and $R=$ $6\left(2 H^{2}+\dot{H}\right)$. In particular, we examine the equation of state for dark energy, given by

$$
\begin{aligned}
& w_{\mathrm{DE}} \equiv P_{\mathrm{DE}} / \rho_{\mathrm{DE}}, \\
& \rho_{\mathrm{DE}}=\frac{1}{\kappa^{2}}\left[\frac{1}{2}(F R-f)-3 H \dot{F}+3(1-F) H^{2}\right], \\
& P_{\mathrm{DE}}=\frac{1}{\kappa^{2}}\left[-\frac{1}{2}(F R-f)+\ddot{F}+2 H \dot{F}-(1-F)\left(2 \dot{H}+3 H^{2}\right)\right],
\end{aligned}
$$

in the future, i.e., the region with the redshift $z<0$. Furthermore, we investigate the evolution of the cosmological horizon entropy. It has been shown that it is possible to obtain a picture of equilibrium thermodynamics on the apparent horizon in the FLRW background for $f(R)$ gravity due to a suitable redefinition of an energy momentum tensor of the "dark" component that respects a local energy conservation in Ref. [25]. In this picture, the horizon entropy $S=A /(4 G)$ [26] where $A$ is the area of the apparent horizon, is simply expressed as [25]

$$
S=\frac{\pi}{G H^{2}} .
$$

We note that in the context of modified gravity theories including $f(R)$ gravity, a horizon entropy $\hat{S}$ associated with a Noether charge has been proposed by Wald [27], given by [28] $\hat{S}=F(R) A /(4 G)$. 

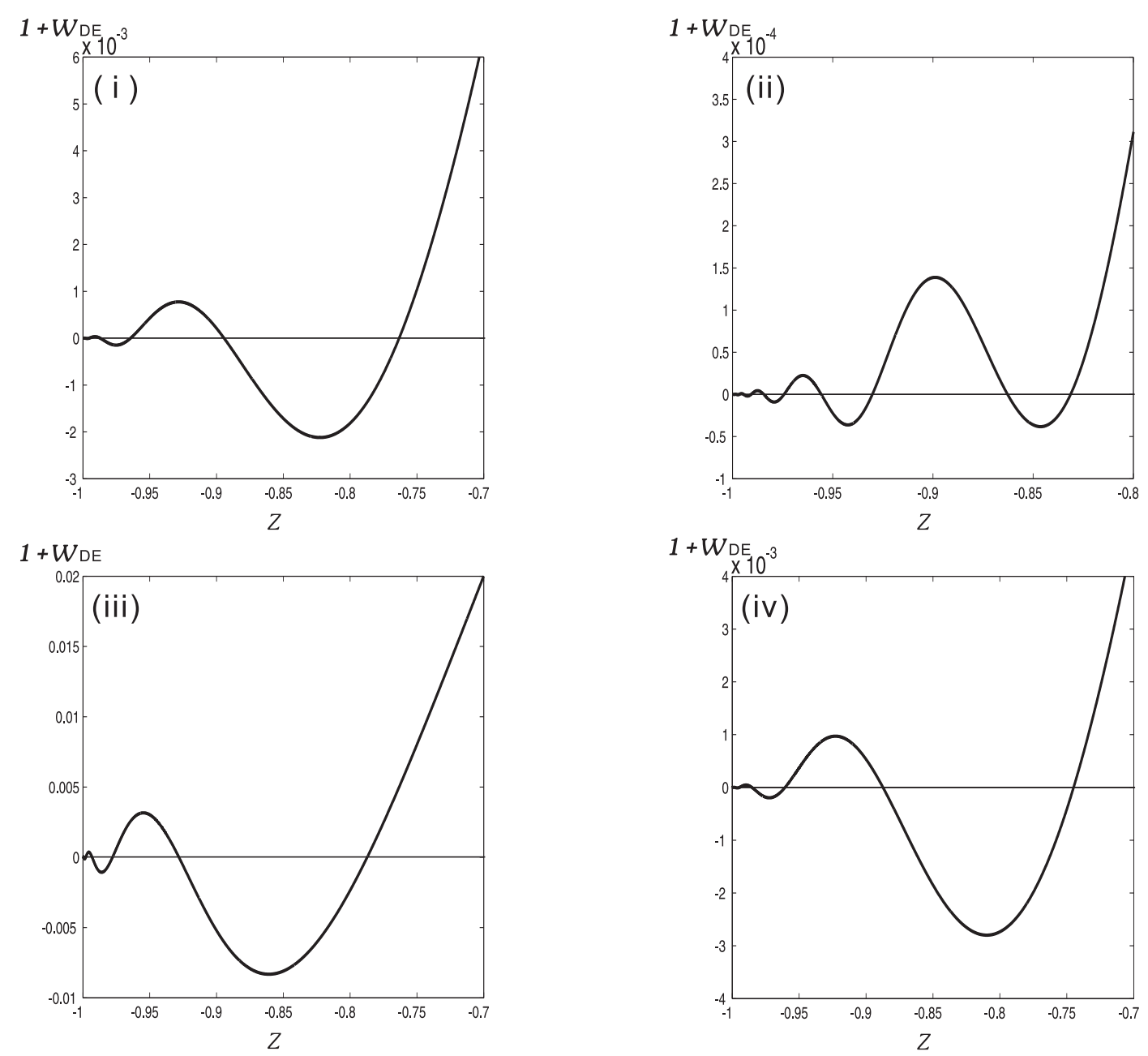

FIG. 1: Future evolutions of $1+w_{\mathrm{DE}}$ as functions of the redshift $z$ in (i) Hu-Sawicki model for $p=1, c_{1}=2$ and $c_{2}=1$, (ii) Starobinsky model for $n=2$ and $\lambda=1.5$, (iii) Tsujikawa model for $\mu=1$ and (iv) the exponential gravity model for $\beta=1.8$, respectively. The thin solid lines show $1+w_{\mathrm{DE}}=0$ (cosmological constant).

In Figs. 1, 2 and 3, we depict the future evolutions of $1+w_{\mathrm{DE}}, \tilde{H} \equiv \bar{H}-\bar{H}_{\mathrm{f}}$ with $\bar{H} \equiv H / H_{0}$ and $\bar{H}_{\mathrm{f}} \equiv H(z=-1) / H_{0}$, and $\tilde{S} \equiv \bar{S}-\bar{S}_{\mathrm{f}}$ with $\bar{S} \equiv S / S_{0}$ and $\bar{S}_{\mathrm{f}} \equiv S(z=-1) / S_{0}$ as functions of the redshift $z \equiv 1 / a-1$ in (i) Hu-Sawicki model for $p=1, c_{1}=2$ and $c_{2}=1$, (ii) Starobinsky model for $n=2$ and $\lambda=1.5$, (iii) Tsujikawa model for $\mu=1$ and (iv) the exponential gravity model for $\beta=1.8$, respectively. Here, $S_{0}=\pi /\left(G H_{0}^{2}\right)$ is the present value of the horizon entropy $S, H_{0}$ is the current Hubble parameter, and the subscript ' $\mathrm{f}$ ' denotes the value at the final stage $z=-1$. Note that the present time is $z=0$ and the future is $-1 \leq z<0$. The parameters used for each model in Figs. 1-3 are the viable ones [29, 30]. Several remarks are as follows: (a) the qualitative results do not 

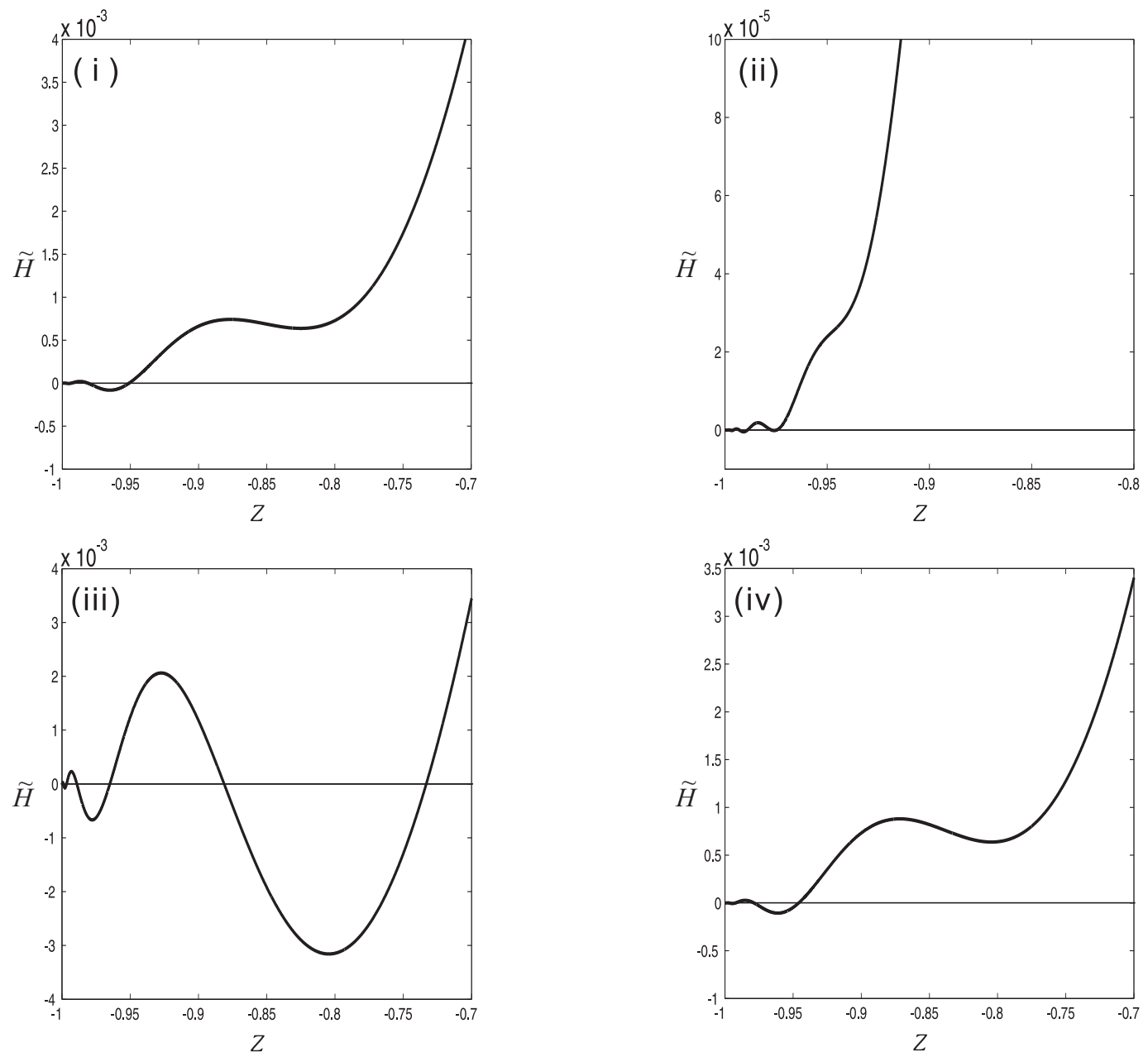

FIG. 2: Future evolutions of $\tilde{H} \equiv \bar{H}-\bar{H}_{\mathrm{f}}$ with $\bar{H} \equiv H / H_{0}$ and $\bar{H}_{\mathrm{f}} \equiv H(z=-1) / H_{0}$ as functions of the redshift $z$. Legend is the same as Fig. 1.

strongly depend on the values of the parameters in each model; (b) the evolutions of the Wald entropy $\hat{S}$ are similar to $S$ [18, 25] in the models of (i)-(iv); and (c) we have studied the Appleby-Battye model [31], which is also a viable $f(R)$ model, and we have found that the numerical results are similar to those in the Starobinsky model of (ii) as expected.

We note that the present values of $w_{\mathrm{DE}}(z=0)$ are $-0.92,-0.97,-0.92$ and -0.93 for the models of (i)-(iv), respectively. These values satisfy the present observational constraints [33]. Moreover, a dimensionless quantity $H^{2} /\left(\kappa^{2} \rho_{\mathrm{m}}^{(0)} / 3\right)$ can be determined through the numerical calculations, where $\rho_{\mathrm{m}}^{(0)}$ is the energy density of non-relativistic matter at the present time. If we use the observational data on the current density parameter of non-relativistic matter $\Omega_{\mathrm{m}}^{(0)} \equiv \rho_{\mathrm{m}}^{(0)} / \rho_{\text {crit }}^{(0)}=0.26$ with $\rho_{\text {crit }}^{(0)}=3 H_{0}^{2} / \kappa^{2}[33]$, we find that the present value of the Hubble parameter $H_{0}=H(z=0)$ is $H_{0}=71 \mathrm{~km} / \mathrm{s} / \mathrm{Mpc}$ [33] for all the models of (i)-(iv). 

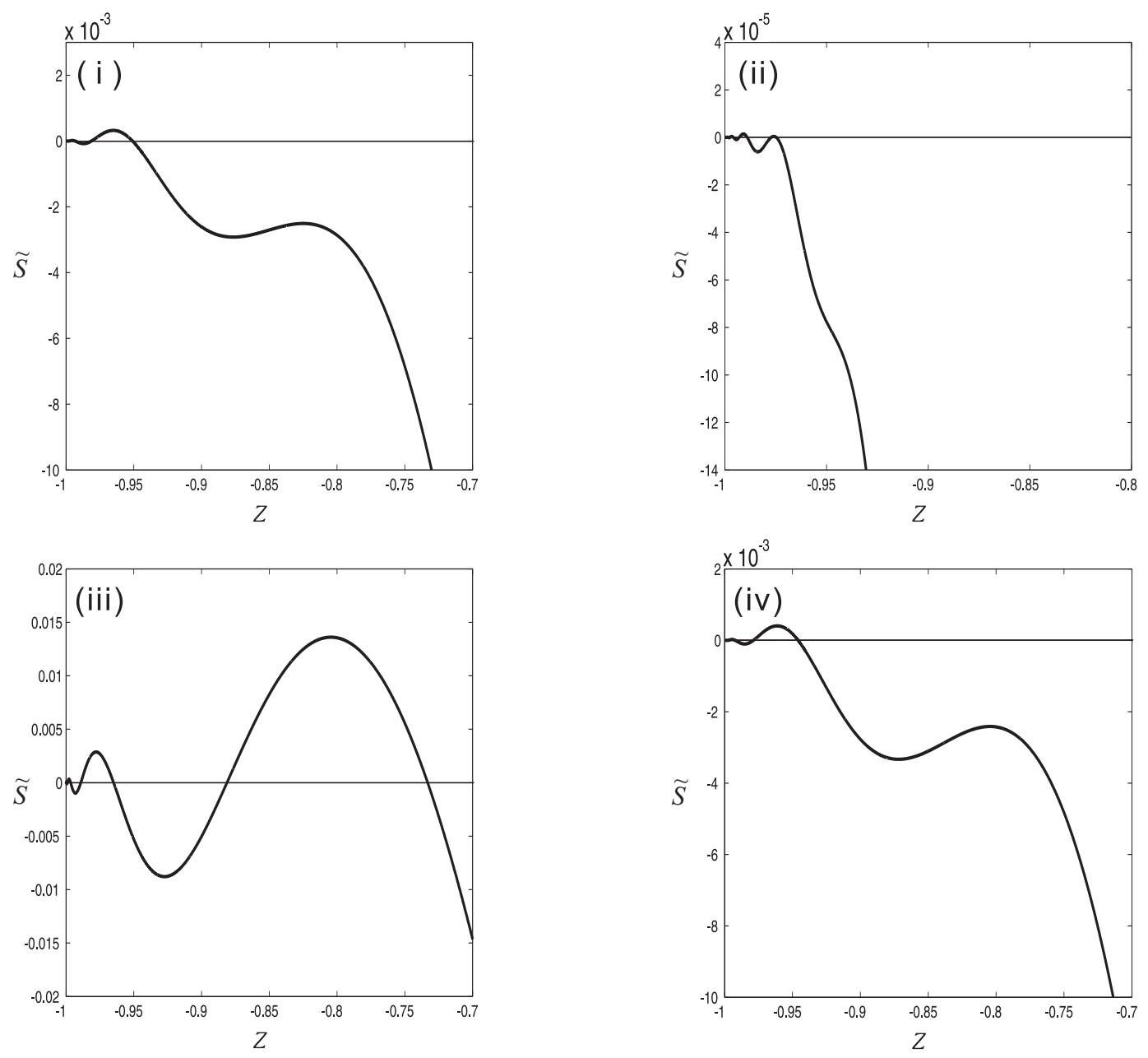

FIG. 3: Future evolutions of $\tilde{S} \equiv \bar{S}-\bar{S}_{\mathrm{f}}$ with $\bar{S} \equiv S / S_{0}$ and $\bar{S}_{\mathrm{f}} \equiv S(z=-1) / S_{0}$ as functions of the redshift $z$. Legend is the same as Fig. 1.

Furthermore, $\left(\bar{H}_{\mathrm{f}}, \bar{S}_{\mathrm{f}}\right)=(0.80,1.6),(0.85,1.4),(0.78,1.7)$ and $(0.81,1.5)$, for the models of (i)-(iv), respectively, where $\bar{H}_{\mathrm{f}} \equiv H(z=-1) / H_{0}$ and $\bar{S}_{\mathrm{f}} \equiv S(z=-1) / S_{0}$.

It is clear from Figs. $1-3$ that in the future $(-1 \leq z \lesssim-0.74)$, the crossings of the phantom divide are the generic feature for all the existing viable $f(R)$ models. By writing the first future crossing of the phantom divide and the first sign change of $\dot{H}$ from negative to positive as $z=z_{\text {cross }}$ and $z=z_{\mathrm{p}}$, respectively, we find that $\left(z_{\text {cross }}, z_{\mathrm{p}}\right)_{\alpha}=(-0.76,-0.82)_{i}$, $(-0.83,-0.98)_{i i},(-0.79,-0.80)_{i i i}$ and $(-0.74,-0.80)_{i v}$, where the subscript $\alpha$ represents the $\alpha$ th viable model. The values of the ratio $\Xi \equiv \Omega_{\mathrm{m}} / \Omega_{\mathrm{DE}}$ at $z=z_{\text {cross }}$ and $z=z_{\mathrm{p}}$ are $\left(\Xi\left(z=z_{\text {cross }}\right), \Xi\left(z=z_{\mathrm{p}}\right)\right)_{\alpha}=\left(5.2 \times 10^{-3}, 2.1 \times 10^{-3}\right)_{i},\left(1.7 \times 10^{-3}, 4.8 \times 10^{-6}\right)_{i i},(4.1 \times$ $\left.10^{-3}, 3.1 \times 10^{-3}\right)_{i i i}$ and $\left(6.2 \times 10^{-3}, 2.8 \times 10^{-3}\right)_{i v}$, where $\Omega_{\mathrm{DE}} \equiv \rho_{\mathrm{DE}} / \rho_{\text {crit }}^{(0)}$ and $\Omega_{\mathrm{m}} \equiv \rho_{\mathrm{m}} / \rho_{\text {crit }}^{(0)}$ are the density parameters of dark energy and non-relativistic matter (cold dark matter 
and baryon), respectively. As $z$ decreases $(-1 \leq z \lesssim-0.90)$, dark energy becomes much more dominant over non-relativistic matter $\left(\Xi=\Omega_{\mathrm{m}} / \Omega_{\mathrm{DE}} \lesssim 10^{-5}\right)$. As a result, one has $w_{\mathrm{DE}} \approx w_{\mathrm{eff}} \equiv-1-2 \dot{H} /\left(3 H^{2}\right)=P_{\mathrm{tot}} / \rho_{\mathrm{tot}}$, where $w_{\mathrm{eff}}$ is the effective equation of state for the universe, and $\rho_{\text {tot }} \equiv \rho_{\mathrm{DE}}+\rho_{\mathrm{m}}+\rho_{\mathrm{r}}$ and $P_{\mathrm{tot}} \equiv P_{\mathrm{DE}}+P_{\mathrm{r}}$ are the total energy density and pressure of the universe, respectively. Here, $\rho_{\mathrm{m}(\mathrm{r})}$ and $P_{\mathrm{r}}$ are the energy density of nonrelativistic matter (radiation) and the pressure of radiation, respectively. The physical reason why the crossing of the phantom divide appears in the farther future $(-1 \leq z \lesssim-0.90)$ is that the sign of $\dot{H}$ changes from negative to positive due to the dominance of dark energy over non-relativistic matter. As $w_{\mathrm{DE}} \approx w_{\text {eff }}$ in the farther future, $w_{\mathrm{DE}}$ oscillates around the phantom divide line $w_{\mathrm{DE}}=-1$ because the sign of $\dot{H}$ changes and consequently multiple crossings can be realized. We remark that since $S \propto H^{-2}$, the oscillating behavior of $S$ comes from that of $H$. However, it should be emphasized that although $S$ decreases in some regions, the second law of thermodynamics in $f(R)$ gravity can be always satisfied because $S$ is the cosmological horizon entropy and it is not the total entropy of the universe including the entropy of generic matter. It has been shown that the second law of thermodynamics can be verified in both phantom and non-phantom phases for the same temperature of the universe outside and inside the apparent horizon in Ref. [32].

Finally, we mention that in our numerical calculations, we have taken the initial conditions of $z_{0}=8.0,8.0,3.0$ and 3.5 for the models of (i)-(iv) at $z=z_{0}$, respectively, so that $R F^{\prime}\left(z=z_{0}\right) \sim 10^{-13}$ with $F^{\prime}=d F / d R$, to ensure that they can be all close enough to the $\Lambda$ CDM model with $R F^{\prime}=0$. We note that in order to save the calculation time, the different values of $z_{0}$ mainly reflect the forms of the models, i.e., the power-law types of (i) and (ii) and the exponential ones of (iii) and (iv). It is clear that the smaller $R F^{\prime}\left(z=z_{0}\right)$ is, the closer the model to $\Lambda$ CDM. However, the cut off of $R F^{\prime}\left(z=z_{0}\right) \sim 10^{-13}$ is assumed to evade the divergence of the calculations in the computing program. We have also checked the results under the initial conditions with $R, f, F$, and $F^{\prime}$ as the $\Lambda$ CDM values at the same redshift up to $z_{0}=4.0$ in the models of (i)-(iv) and found that they are qualitatively similar. We remark that at $z=z_{0}, w_{\mathrm{DE}}=-1$.

Since $R / R_{\mathrm{c}} \gg 1$ in the high $z$ regime $\left(z \simeq z_{0}\right)$, the value of the combination $\gamma R_{\mathrm{c}}$ is set as $\gamma R_{\mathrm{c}} \simeq 18 H_{0}^{2} \Omega_{\mathrm{m}}^{(0)}$, where $\left(\gamma, R_{\mathrm{c}}\right)$ corresponds to $\left(c_{1}, R_{\mathrm{HS}}\right),\left(\lambda, R_{\mathrm{S}}\right),\left(\mu, R_{\mathrm{T}}\right)$ and $\left(\beta, R_{\mathrm{E}}\right)$ for (i) Hu-Sawicki $\left(c_{2}=1\right.$ ), (ii) Starobinsky, (iii) Tsujikawa and (iv) the exponential gravity models, respectively, and $\Omega_{\mathrm{m}}^{(0)} \equiv \rho_{\mathrm{m}}^{(0)} / \rho_{\text {crit }}^{(0)}=0.26$ [33]. The reason is as follows. In the high 
$z$ regime $\left(z \simeq z_{0}\right), R / R_{\mathrm{c}} \gg 1$, in which $f(R)$ gravity has to be very close to the $\Lambda \mathrm{CDM}$ model, $\gamma R_{\mathrm{c}} \simeq 2 \Lambda=2 \kappa^{2}\left(\rho_{\mathrm{DE}} / \rho_{\mathrm{m}}^{(0)}\right)\left(\rho_{\mathrm{m}}^{(0)} / \rho_{\text {crit }}^{(0)}\right) \rho_{\text {crit }}^{(0)}=6\left(\rho_{\mathrm{DE}} / \rho_{\mathrm{m}}^{(0)}\right) H_{0}^{2} \Omega_{\mathrm{m}}^{(0)}$, where $\Lambda$ is the effective cosmological constant in the limit of $R / R_{\mathrm{c}} \gg 1$. As an initial condition, we take $\left(\rho_{\mathrm{DE}} / \rho_{\mathrm{m}}^{(0)}\right)=3.0$ [18]. Thus, we obtain $\gamma R_{\mathrm{c}} \simeq 18 H_{0}^{2} \Omega_{\mathrm{m}}^{(0)}$.

Since the initial value at $z=z_{0}$ of the variable $y_{H} \equiv \rho_{\mathrm{DE}} / \rho_{\mathrm{m}}^{(0)}$ introduced in Ref. [18] is an arbitrary one, we have chosen it by using the observational data [33] at the present time as $y_{H}\left(z=z_{0}\right) \simeq \Omega_{\mathrm{DE}}^{(0)} / \Omega_{\mathrm{m}}^{(0)} \simeq 3.0$. The physical reason is as follows. By examining the cosmological evolutions of $y_{H}$ and $w_{\mathrm{DE}}$ as functions of the redshift $z$ for the models, we have found that $y_{H}(z=0)$ is close to its initial value of $y_{H}\left(z=z_{0}\right)$. This is because in the higher $z$ regime, the universe is in the phantom phase $\left(w_{\mathrm{DE}}<-1\right)$ and therefore, $\rho_{\mathrm{DE}}$ and $y_{H}$ increase (since $y_{H} \propto \rho_{\mathrm{DE}}$ ), whereas in the lower $z$ regime, the universe is in the non-phantom (quintessence) phase $\left(w_{\mathrm{DE}}>-1\right)$ and hence they decrease. Consequently, the above two effects cancel out. To check our numerical results, by using the way in, e.g., Ref. [30], we have made another numerical calculation for the model of (iv) as follows. First, by taking a testing value of $\gamma R_{\mathrm{c}}$ at an initial value of $z_{0}$, we analyze the cosmological evolution numerically. Next, we find the adequate value of $z_{0}$ so that the current value of $y_{H}(z=0)$ can be equal to the present observational value of $\rho_{\mathrm{DE}}^{(0)} / \rho_{\mathrm{m}}^{(0)}=\left(1-\Omega_{\mathrm{m}}^{(0)}\right) / \Omega_{\mathrm{m}}^{(0)}=2.85$, where $\rho_{\mathrm{DE}}^{(0)}$ is the present energy density of dark energy. We also obtain the initial value of $y_{H}\left(z=z_{0}\right)$ and the value of $R_{\mathrm{c}} \simeq 6 \gamma^{-1} y_{H}\left(z=z_{0}\right) \bar{m}^{2}$ with $\bar{m}^{2}=H_{0}^{2} \Omega_{\mathrm{m}}^{(0)}$. As a result, we have found that $y_{H}\left(z=z_{0}\right)=2.72$ with $z_{0}=3.0$ for $y_{H}(z=0)=2.85$. Furthermore, we have confirmed that the obtained results with the above method are qualitatively similar to the ones shown in Figs. 1-3. Clearly, our results are not sensitive to the initial values of $z_{0}$ and $y_{H}\left(z=z_{0}\right)$. We note that the cosmological evolution of $w_{\mathrm{DE}}$ as a function of the redshift $z$ for the model of (iv) is given in Fig. 5 of Ref. [18]. On the other hand, the initial condition of $d y_{H} / d \ln a\left(z=z_{0}\right)=0$ is due to that the $f(R)$ gravity models at $z=z_{0}$ should be very close to the $\Lambda$ CDM model, in which $d y_{H} / d \ln a=0$.

\section{CONCLUSIONS}

In the present paper, we have explored the future evolution of $w_{\mathrm{DE}}$ in the viable $f(R)$ gravity models and explicitly shown that in the future the crossings of the phantom divide are the generic feature in these models. We have also investigated the future evolution of 
the cosmological horizon entropy and demonstrated that the cosmological horizon entropy oscillates with time because the Hubble parameter also does. The new cosmological ingredient obtained in this study is that in the future the sign of $\dot{H}$ changes from negative to positive due to the dominance of dark energy over non-relativistic matter. This is a common physical phenomena to the existing viable $f(R)$ models and thus it is one of the peculiar properties of $f(R)$ gravity models characterizing the deviation from the $\Lambda$ CDM model.

\section{Acknowledgments}

The work is supported in part by the National Science Council of R.O.C. under Grant \#s: NSC-95-2112-M-007-059-MY3 and NSC-98-2112-M-007-008-MY3 and National Tsing Hua University under the Boost Program and Grant \#: 99N2539E1.

[1] S. Perlmutter et al. [SNCP Collaboration], Astrophys. J. 517, 565 (1999); A. G. Riess et al. [SNST Collaboration], Astron. J. 116, 1009 (1998).

[2] D. N. Spergel et al. [WMAP Collaboration], ibid. 170, 377 (2007).

[3] M. Tegmark et al., Phys. Rev. D 69, 103501 (2004); D. J. Eisenstein et al., Astrophys. J. 633, $560(2005)$.

[4] B. Jain and A. Taylor, Phys. Rev. Lett. 91, 141302 (2003).

[5] For a review on dark energy, see E. J. Copeland, M. Sami and S. Tsujikawa, Int. J. Mod. Phys. D 15, 1753 (2006).

[6] S. Nojiri and S. D. Odintsov, eConf C0602061, 06 (2006) [Int. J. Geom. Meth. Mod. Phys. 4, 115 (2007)] arXiv:hep-th/0601213].

[7] T. P. Sotiriou and V. Faraoni, Rev. Mod. Phys. 82, 451 (2010).

[8] A. De Felice and S. Tsujikawa, arXiv:1002.4928 [gr-qc].

[9] S. Nojiri and S. D. Odintsov, Phys. Rev. D 68, 123512 (2003); A. D. Dolgov and M. Kawasaki, Phys. Lett. B 573, 1 (2003); V. Faraoni, Phys. Rev. D 74, 104017 (2006); Y. S. Song, W. Hu and I. Sawicki, ibid. 75, 044004 (2007).

[10] V. Muller, H. J. Schmidt and A. A. Starobinsky, Phys. Lett. B 202, 198 (1988); V. Faraoni and S. Nadeau, Phys. Rev. D 72, 124005 (2005); L. Amendola, R. Gannouji, D. Polarski and 
S. Tsujikawa, ibid. 75, 083504 (2007).

[11] T. Chiba, Phys. Lett. B 575, 1 (2003); T. Chiba, T. L. Smith and A. L. Erickcek, Phys. Rev. D 75, 124014 (2007).

[12] W. Hu and I. Sawicki, Phys. Rev. D 76, 064004 (2007).

[13] A. A. Starobinsky, JETP Lett. 86, 157 (2007).

[14] S. Tsujikawa, Phys. Rev. D 77, 023507 (2008).

[15] P. Zhang, Phys. Rev. D 73, 123504 (2006); B. Li and M. C. Chu, ibid. 74, 104010 (2006); R. Bean, D. Bernat, L. Pogosian, A. Silvestri and M. Trodden, ibid. 75, 064020 (2007); P. J. Zhang, ibid. 76, 024007 (2007); P. Zhang, M. Liguori, R. Bean and S. Dodelson, Phys. Rev. Lett. 99, 141302 (2007); G. Calcagni and G. Nardelli, arXiv:1004.5144 [hep-th].

[16] G. Cognola, E. Elizalde, S. Nojiri, S. D. Odintsov, L. Sebastiani and S. Zerbini, Phys. Rev. D 77, 046009 (2008).

[17] E. V. Linder, Phys. Rev. D 80, 123528 (2009).

[18] K. Bamba, C. Q. Geng and C. C. Lee, JCAP 1008, 021 (2010) arXiv:1005.4574 [astro-ph.CO].

[19] S. Nojiri and S. D. Odintsov, Phys. Lett. B 657, 238 (2007); Phys. Rev. D 77, 026007 (2008); AIP Conf. Proc. 1241, 1094 (2010) arXiv:0910.1464 [hep-th]].

[20] U. Alam, V. Sahni and A. A. Starobinsky, JCAP 0406, 008 (2004); 0702, 011 (2007); S. Nesseris and L. Perivolaropoulos, ibid. 0701, 018 (2007); P. U. Wu and H. W. Yu, Phys. Lett. B 643, 315 (2006); H. K. Jassal, J. S. Bagla and T. Padmanabhan, arXiv:astro-ph/0601389.

[21] R. R. Caldwell, Phys. Lett. B 545, 23 (2002); B. Feng, X. L. Wang and X. M. Zhang, ibid. 607, 35 (2005); Z. K. Guo, Y. S. Piao, X. M. Zhang and Y. Z. Zhang, ibid. 608, 177 (2005).

[22] M. Martinelli, A. Melchiorri and L. Amendola, Phys. Rev. D 79, 123516 (2009); H. Motohashi, A. A. Starobinsky and J. Yokoyama, arXiv:1002.1141 [astro-ph.CO]; arXiv:1005.1171 [astroph.CO].

[23] M. C. B. Abdalla, S. Nojiri and S. D. Odintsov, Class. Quant. Grav. 22, L35 (2005); I. H. Brevik, Int. J. Mod. Phys. D 15, 767 (2006); Gen. Rel. Grav. 38, 1317 (2006); L. Amendola and S. Tsujikawa, Phys. Lett. B 660, 125 (2008); K. Bamba, C. Q. Geng, S. Nojiri and S. D. Odintsov, Phys. Rev. D 79, 083014 (2009); Mod. Phys. Lett. A 25, 900 (2010); K. Bamba and C. Q. Geng, Phys. Lett. B 679, 282 (2009); K. Bamba, arXiv:0904.2655 [gr-qc]; K. Bamba, S. Nojiri and S. D. Odintsov, JCAP 0810, 045 (2008); Y. Bisabr, arXiv:1005.5679 [gr-qc].

[24] K. Bamba and C. Q. Geng, Prog. Theor. Phys. 122, 1267 (2009). 
[25] K. Bamba, C. Q. Geng and S. Tsujikawa, Phys. Lett. B 688, 101 (2010).

[26] J. M. Bardeen, B. Carter and S. W. Hawking, Commun. Math. Phys. 31, 161 (1973); J. D. Bekenstein, Phys. Rev. D 7, 2333 (1973); S. W. Hawking, Commun. Math. Phys. 43, 199 (1975) [Erratum-ibid. 46, 206 (1976)].

[27] R. M. Wald, Phys. Rev. D 48, 3427 (1993); V. Iyer and R. M. Wald, ibid. 50, 846 (1994).

[28] T. Jacobson, G. Kang and R. C. Myers, Phys. Rev. D 49, 6587 (1994); G. Cognola, E. Elizalde, S. Nojiri, S. D. Odintsov and S. Zerbini, JCAP 0502, 010 (2005).

[29] S. Capozziello and S. Tsujikawa, Phys. Rev. D 77, 107501 (2008); A. Ali, R. Gannouji, M. Sami and A. A. Sen, ibid. 81, 104029 (2010).

[30] S. Tsujikawa, R. Gannouji, B. Moraes and D. Polarski, Phys. Rev. D 80, 084044 (2009).

[31] S. A. Appleby and R. A. Battye, Phys. Lett. B 654, 7 (2007); S. Appleby, R. Battye and A. Starobinsky, JCAP 1006, 005 (2010).

[32] K. Bamba and C. Q. Geng, JCAP 1006, 014 (2010) arXiv:1005.5234 [gr-qc]].

[33] E. Komatsu et al., arXiv:1001.4538 [astro-ph.CO]. 